\title{
The Bragg Symposium 1970
}

A Symposium entitled X-ray Analysis - Past, Present, and Future was held at the Royal Institution on April $1-3$ to celebrate the 80th birthday of Sir Lawrence Bragg who was born on 31 March 1890. The Symposium was sponsored by the Royal Society, the Institute of Physics and the Physical Society, and the International Union of Crystallography. It was attended by almost 400 active participants, of whom about one third were from overseas, and by nearly 100 accompanying members. Apart from the scientific sessions, a reception was held at the rooms of the Royal Society on the evening of 1 April, and the Symposium Dinner at Imperial College on 2 April. There was also on display in the Royal Institution and in the rooms of the Royal Society a fascinating selection of papers and correspondence relating to the life and work of Sir Lawrence Bragg.

The scientific sessions started with the Bragg Lecture. The remainder of the programme consisted of invited and contributed papers on four topics: Minerals; Metals; Methods of Analysis; and Big Molecules.

\section{Minerals}

Mineralogy and mineralogists played a special role in the early days of the development of X-ray crystallography. Mineral collections were the source of crystals of chemically simple compounds used by the Braggs to test their theories of X-ray diffraction. In retrospect, this cooperation from mineralogy was the very least that it could offer for what was to emerge as a profoundly improved classification and understanding of the mineral kingdom resulting from the many structure determinations effected by W. L. Bragg, his first students and later generations of crystallographers.

One of Bragg's early co-workers on silicate structures was B. E. Warren (Massachusetts Institute of Technology). Very appropriately, the sessions of the Symposium were preceded by the Fourth Bragg lecture, given by Warren, entitled The $X$-ray Analysis of Glass Structures. Warren showed how, in the study of silicon and boron oxide glasses, two new techniques have given vastly improved results. One of the biggest difficulties hitherto was the distinction between coherent and Compton scattering by glasses, and this has been overcome by counting fluorescence radiation produced intensely only from the coherent fraction by a specially chosen scatterer ( $\mathrm{R} h$ radiation, Mo scatterer). A further marked improvement is obtained by the use of 'pair function distributions' (a concept first developed by C. Finbak and taken further by Warren) which eliminates many of the approximations involved in earlier work. When applied together, these methods can yield very good correspondence between observed and calculated pair function curves for a correct model chosen by trial and error. Professor Warren thought that much of the old X-ray work on glasses could be profitably redone using the new techniques.

The most abundant mineral in the earth's crust is feldspar. Its basic structure appeared simple, once determined by W. H. Taylor, but it was soon apparent also that the details of variation among different kinds of feldspar were extremely complex. H. D. Megaw (Cambridge), in her invited paper Topology and Tetrahedra in Feldspars, emphasized the simple rather than complex. Regarding the framework as made up from mechanically hinged ideal and rigid tetrahedra, she showed that the variations in cell parameters among the feldspars were influenced far more by the tilts of tetrahedra than by order-disorder effects among the cations. Megaw went on to show that similar mechanical models can be used fruitfully to help understand features of the structures of the silica minerals.

One of the more complex aspects of feldspar structures was dealt with in the contribution by M. Czank, F. Laves and H. Schulz (E. T. H., Zürich) on The Domains of Anorthite. On the basis of nuclear magnetic resonance results, calcic feldspars are pictured as having an 'out of step domain' texture. Changes that occur with temperature were likened to order-disorder changes with a critical temperature of $230^{\circ} \mathrm{C}$, below which the structure is primitive and above almost completely body-centred. The changes involved were described as to some extent displacive transformations, but also as comparable in some ways with ferroelectric transitions.

The work of the 'Bragg Manchester School' of crystallographers ranged over the principal rock-forming silicates, the olivines, pyroxenes, amphiboles, micas, feldspars, feldspathoids and zeolites. In these, $\mathrm{SiO}_{4}$ or $\mathrm{AlO}_{4}$ tetrahedra are arranged (singly or in pairs, rings, chains, etc.) in what might be called 'Bragg configurations'. It would be surprising, however, if these were the only configurations to occur, and indeed less common silicates exhibit rather different arrangements. The structures of many such silicates have been determined by N. V. Belov (Academy of Sciences, USSR) and his co-workers. Belov, in New Aspects of the Crystal Chemistry of Silicates, illustrated a number of the more unusual silicate structures, charmingly suggesting that what he has been calling Chapter 2 of the classification of silicates (complementing Bragg's 'Chapter $\left.1^{\prime}\right)$, is now better thought of as part 2 of one long chapter of silicate structures following the pioneering work of Bragg.

Many of the structures determined by the Bragg School were of the more common mineral oxides. An invited paper, Structural Hierarchies among Minerals Containing Oxygen, was presented by P. B. Moore (Chicago), in which he explained his attempt to system- 
atize all possible structures based upon octahedra of oxygen atoms. For the phosphates, arsenates and sulphates of the first transition series of elements, the different octahedral clusters which can occur have been explored by use of graph theory, nodes corresponding to octahedral centres and branches corresponding to the linking units between nodes. For each cluster, topological isomers, geometrical isomers and stereoisomers need to be considered. Moore hoped that such an exploration would lead to a better appreciation of the relationships between mineral structures which are not easily discerned from present methods of mineral classification.

The next paper was one by M. L. Huggins (California) entitled The Structon Theory: Stabilities of Different Structon Types. He discussed the coordination numbers for oxygen around $\mathrm{Si}, \mathrm{B}$ and $\mathrm{Al}$, and in particular the bridging of oxygen atoms between like and unlike atoms.

The sessions on minerals concluded with a paper by C. Frondel (Harvard) on The Mineralogy of Lunar Materials. He indicated that the major minerals which form the Apollo 11 and Apollo 12 lunar rocks are the common terrestrial minerals, pyroxene, feldspar, ilmenite and olivine, but the proportions of these minerals, and the chemistry of the rocks and minerals, set the lunar rocks apart from anything else of which we have had experience hitherto.

\section{Metals}

The proceedings of the sessions on metals may be introduced by summarizing the remarks made by Sir Lawrence Bragg at the end of the sessions. Sir Lawrence recalled the first metal structure to be investigated by $\mathrm{X}$-ray diffraction, that of copper, the crystal used being 'borrowed' from the mineralogical collection in Cambridge, and reminded us that these early studies led to the important concept of atomic size. He remarked that the application of X-ray analysis to more complicated systems was typified by the determination of the structure of $\gamma$-brass by Bradley and Lipson in Manchester and that this structure, in common with many alloys, is so flexible that it can be formed from many combinations of elements in widely differing proportions. The final important development recalled by Sir Lawrence was the recognition of the order-disorder transition in alloys and the development of the Bragg-Williams theory.

The concept of atomic size provides a link between the first two invited papers Structural Principles of Complex Intermetallic Compounds by S. Samson (California Institute of Technology) and Alloys of Transition Elements having Unfilled $p$-Levels by S. Rundquist (Uppsala). Samson reviewed 56 years of X-ray diffraction studies of alloy structures in terms of the closepacking of spheres. He showed how a slight distortion of the twelvefold coordination polyhedron of copper gives an icosahedron which enables twelve spheres to be packed around a slightly smaller one and demonstrated the construction of the 16-fold Friauf polyhedron as a packing of twelve small and four large spheres around a central large sphere. Samson then went on to show how these two and a few further coordination polyhedra can be used to build up the structures of very many alloys, and demonstrated the usefulness of this approach by showing how a study of the packing of these polyhedra had enabled the very complex structures of $\mathrm{Mg}_{2} \mathrm{Al}_{3}$ and $\mathrm{Cu}_{4} \mathrm{Cd}_{3}$ to be determined. In response to a question about the validity of the structures derived for these compounds, Samson emphasized that the method was useful for structure determination, since it enabled size considerations to be satisfied and gave trial structures which were capable of subsequent refinements.

Rundquist was also concerned with atomic sizes. $\mathrm{He}$ defined a $p$-element as one whose valence shell contains unfilled $p$-levels. Compounds formed between $p$-elements and transition elements are found to be metallic and to depend rather little on the identity of the $p$-element so long as the transition metal content is high. At small radius ratios $\left(r_{p} / r_{t}<0.59\right)$ they form interstitial alloys and at larger radius-ratios complex structures are favoured. Rundquist went on to consider the common features of such complex structures and showed that many of them could be understood in terms of the coordination polyhedron of metal atoms around a $p$-atom. The most important of these is the triangular prism which may have from one to three additional metal atoms outside the quadrilateral faces. $\mathrm{He}$ illustrated the relationship between the structures of $\mathrm{Ni}_{2} \mathrm{In}, \mathrm{Co}_{2} \mathrm{Si}$ and $\mathrm{Co}_{2} \mathrm{P}$ and pointed out that there are 150 known compounds with one of these three types of structure. Some further information about compounds between transition metals and $p$-elements was contained in The Electron Configuration in $\mathrm{Fe}_{2} \mathrm{~B}$ from $X$-ray and Neutron Scattering Measurements by P. J. Brown and J. L. Cox (Cambridge). The two techniques provide complementary evidence about the electronic states occupied in the alloy and suggest that the valence electrons of boron are non-localized and do not enter the iron $3 d$-band as has been suggested in the past.

The invited paper Ferroelasticity, Memory Effects, and Phase Transformations in Crystalline Solids by D. S. Lieberman (Illinois) focused attention on transitions in alloy structures. By means of a series of demonstrations, fully in keeping with the traditions of the Royal Institution, Lieberman demonstrated a ferroelastic transition in a gold-cadmium alloy and suggested a mechanism by which it might take place. He also discussed a memory effect in the transition of a titanium-nickel alloy. This alloy will return to the same shape in the high temperature form regardless of how it is deformed below the transition. The demonstration of this property, in which a wire on heating assumed the shape of a $\mathrm{B}$, provided an additional small tribute to Sir Lawrence. 


\section{Methods of analysis}

These sessions consisted of four invited and two contributed papers. C. A. Taylor (Cardiff) in The Development of Optical Transform Techniques pointed out that, although the method is of little value for the solution of the phase problem of complex structures, it nevertheless remains of great importance for teaching and understanding the basic physics of diffraction. The gas-phase laser can be used to produce images of an intensity sufficient to be readily visible in lecture demonstrations. Also, because of the fundamental nature of the process, experiments on optical transforms have generated invaluable additional X-ray diffraction apparatus, such as the precession camera.

P. Tollin (Dundee) in Reciprocal Space Methods for Patterson Function Interpretation considered the problem of using the Patterson function to solve a structure containing a known chemical grouping. He emphasized that the use of the sum function as a criterion of fit enables the subject to be considered in reciprocal space, and illustrated his points by reference to the crystal structure of inosine as well as to fibres.

J. Karle (Naval Research Laboratory, Washington) whose persistent work has been largely responsible for the widespread acceptance of direct methods gave a brief review of the fundamental sign relations in Direct Methods and Structures of Unknown Formula. He illustrated the power of the method by mentioning the crystal structures of a variety of substances that have been investigated in his and other laboratories. He stressed that, particularly in the triclinic system, if the initial application of direct methods is unsuccessful, a satisfactory solution can usually be obtained by going back and starting again with a different set of origindetermining and symbolic phases. Moreover, in noncentrosymmetric crystals, it may well happen that direct methods reveal only a portion of the structure. It is then often most economical to use this partial structure together with the tangent formula to obtain a complete solution.

P. Main (York) in Towards the Automatic Determination of Non-centrosymmetric Crystal Structures discussed a full set of computer programs that attempts to make the whole process of phase determination by direct methods automatic. He was particularly concerned with the stage, normally performed by hand, in which the $\sum_{1}$ formula is used to determine the signs of centric reflexions which are structure invariants. The procedure involves the successive elimination of planes whose phases are considered to be unreliable, the criterion being the sum of the magnitudes of the individual $\sum_{2}$ contributions. In the end a small set of highly consistent planes is obtained, and these are used as the starting point for phase determination by the tangent formula, care being taken to assign a weight to each plane according to the same criterion.

E. B. Fleischer (Chicago) in The Structure of a Synthetic Steroid described how direct methods initially failed to provide a solution of the structure of this molecule of formula $\mathrm{C}_{18} \mathrm{H}_{26} \mathrm{O}_{2}$, which crystallizes in the space group $P \overline{1}$. After several attempts a fused fiveand six-membered ring was recognized, but no progress could be made by Fourier refinement in the space group $P \overline{1}$. In order to overcome this difficulty, the space group was relaxed to $P 1$, and the fragment used to calculate phases which were further refined by the tangent formula. Fourier refinement was then carried out in space group $P 1$ until all but six of the forty carbon and oxygen atoms had been located. The centre of symmetry was then introduced at the appropriate point and refinement completed in the space group $P \overline{1}$.

W. D. S. Motherwell (Cambridge) concluded the session by describing in Adenosine Triphosphate - Determination of the Structure by Direct Methods how the structure of ATP, which contains two formula weights of composition $\mathrm{C}_{10} \mathrm{H}_{14} \mathrm{~N}_{5} \mathrm{Na}_{2} \mathrm{O}_{13} \mathrm{P}_{3} .3 \mathrm{H}_{2} \mathrm{O}$ in the asymmetric unit of space group $P 22_{1} 2_{1} 2_{1}$, was obtained. The solution was not straightforward because the availability of only very small crystals and the presence of some degree of disorder restricted the number of reflexions with significant intensities, but an application of the tangent formula led to the correct solution.

\section{Big molecules}

Without exception, the molecules discussed in this session have important functions in living things and the last decade has seen a rapid growth in this area of crystallography. Prior to 1960, Sir Lawrence, as Cavendish Professor of Physics in Cambridge, provided much of the backing and encouragement that was needed to launch this subject successfully, and, following the breakthrough that the structure determinations of myoglobin and haemoglobin represented, he continued at the Royal Institution to promote this work during the period that Phillips and co-workers were determining the structure of lysozyme. During this session Sir Lawrence recalled that he had predicted in 1960 that ten years would elapse before another protein structure was determined and was delighted to have been proved wrong. In the event, more than twenty independent protein structure determinations have now been accomplished and many more are in progress. These studies have brought about the interaction of crystallography and crystallographers with the life sciences, especially evolution, genetics, and enzyme chemistry. Reactions analogous to some of those that occur in living cells may be performed and studied in crystals, and the changes produced by genetic mutation during evolution may be directly related to the structure and function of a protein through the cooperation of biochemists and crystallographers.

These points were very well illustrated by R. E. Dickerson (California Institute of Technology) in Enzymes: Machinery with a History. He described the structure of horse cytochrome-c and its relation to that of the bonito. Cytochrome-c is one of a series of mitochon- 
drial enzymes involved in the conversion of energy from carbohydrates into the high-energy molecule adenosine triphosphate which in turn applies its energy in muscular contraction and other energy requiring processes. Like the globins, this molecule contains a haem group (a porphyrin ring system with a central iron atom), but, unlike the globins, the haem is covalently bound to the protein through sulphur atoms of cysteine side chains. The molecule is smaller than myoglobin and consequently the protein chain enwraps the haem group with relatively little to spare and, perhaps as a result, the molecule is relatively intolerant of mutations, with a mean mutation rate estimated at one successful amino acid change per $21 \times 10^{6}$ years. The majority of the amino acid side chains either recognizably play a part in binding the haem in a hydrophobic environment, or they are involved in the solvation of the molecule as a whole. Myoglobin and haemoglobin, with their larger amounts of seemingly superfluous material, have mutation rates which are estimated to be three times as fast.

R. Huber (Max Planck Institute, Munich) in The Structure of an Insect Haemoglobin presented the results of his studies of erythrocruorin obtained from Chironomus thumi. This protein forms an interesting comparison with mammalian haemoglobins and myoglobins because the evolution of mammals and insects is believed to have diverged some $750 \times 10^{6}$ years ago. The erythrocruorin protein chain is some 17 residues shorter than sperm whale myoglobin, and about 10 shorter than mammalian haemoglobin chains. Nevertheless the insect protein has essentially the same structure as the mammalian globins, the differences being but variations on a theme. The differences are interesting, however, and illustrate the relatively high tolerance to mutations of this type of molecule. At the $\mathrm{AB}$ corner for example (i.e. the region where the protein chain discontinues the regular formation of the first $\alpha$-helix and initiates a second helix in a new direction) the insect protein has several residues less than the mammalian proteins so the chain cuts the corner and also disturbs the position of the second helix. This is a large mutation, yet the protein is viable because the alterations are some distance from the haem group. Close to the haem group there are many differences of side chains but little alteration to the course of the main chain.

J. Greer (Cambridge) in X-ray Studies of Mutant Human Haemoglobins described similar work on related molecules arising in cases of hereditary blood diseases. He showed how much more minor mutations, involving single amino acid side chains, can seriously impair the functioning of the molecule if these alterations occur at more vital points.

In addition to these somewhat related papers additional structures were described in The Crystal and Molecular Structure of Insulin by M. Vijayan (Oxford) and Lactate Dehydrogenase - $2 \cdot 8 \AA$ Resolution Results by $M$. Adams (Purdue). Insulin is now a well characterized structure in which all the side chains are identified. However, the mechanism of action of this hormone in its biological environment is not yet understood in terms of its structure. Lactate dehydrogenase, on the other hand, is perhaps better understood than insulin and suggestions have already been put forward for the mode of binding of this enzyme's substrate, though a fuller understanding of the molecule must await the determination of its sequence.

Finally L. N. Johnson (Oxford) in Structural Studies on Aldolase described work on aldolase which is still at an early stage.

The Symposium, which was organized largely by Dr W. H. Taylor (Cambridge), was an enormous success, and the fact that Sir Lawrence Bragg was present for every single moment, asking searching questions, making probing comments, giving comprehensive summaries, and delivering light-hearted speeches, suggests that it is none too soon to be thinking about what should be done when he is ninety. 